\title{
What value for the phase IV clinical trials?
}

\author{
Michel Bourin ${ }^{1 *}$ and Abdeslam Chagraoui ${ }^{2}$ \\ ${ }^{1}$ Neurobiology of Mood disorders, University of Nantes, 98 rue Joseph Blanchart 44100 Nantes, France. \\ ${ }^{2}$ Inserm U982, Laboratory of Neuronal and Neuroendocrine Differentiation and Communication, Institute for Research and Innovation in Biomedicine, \\ Normandy University, France; Department of Medical Biochemistry, Rouen University Hospital, Rouen, France
}

Received: June 07, 2016; Accepted: June 18, 2016; Published: July 05, 2016

*Corresponding author: Michel Bourin, Neurobiology of Mood disorders, University of Nantes, 98 rue Joseph Blanchart 44100 Nantes, France; E-mail: michel.bourin@univ-nantes.fr

\begin{abstract}
Phase IV of clinical trials also named post marketing studies or real world trials, are useful to better understand the true efficacy of medicines.

Phase IV could improve scientific knowledge i.e. side effects, use of a drug in special conditions, better precision of the dosage. But some studies are more designed for economic purposes facilitating product promotion.

So there are several kinds of phase IV clinical trials: clinical research, seeding trials, prescription studies or pseudo-trials for commercial purposes.

Keywords: Phase IV clinical trials; Post marketing studies; Real world trials
\end{abstract}

\section{Introduction}

Phases I, II and III are essential steps in the development of a drug for approved registration. For certain drugs, phase IV begins as soon as this approval is obtained, whereas for others it comes after marketing has started and throughout the commercial life of the drug [1].

By definition, phase IV includes all the trials undertaken to study a drug in the strict context of the indications and dosage approved at the time of registration. These phase IV trials are intended to provide better knowledge of the usefulness of the drug for the patient and society. An indication of the advantages of a drug, a more complete collection of epidemiologic data, and a strategy of evaluation adapted to the particular circumstances of each pathology should lead to greater efficacy and utility, better quality of life and improvement of the cost-benefit ratio.

New data needed to improve the medical services offered and assess therapeutic needs require continual observation and evaluation of the drug and its environment from initial marketing to the end of its commercial life. It is obviously in ambulatory practice that a drug can best be observed in its ordinary environment, improved with respect to its indications and precautions for use, and judged (observed and analyzed) in terms of its role in everyday circumstances.

The results of a phase IV clinical trial can give rise to new phase
II and III studies in order to enlarge the indications modify the dosage and elaborate new galenic forms. Any study undertaken to change the conditions in which drug registration was obtained should in fact be considered as a phase III clinical trial.

\section{Usefulness of phase IV clinical trials}

Phase IV clinical trials are essential for two main reasons:

Improving scientific knowledge: The knowledge of a drug is still often incomplete at the end of a phase III trial because the number of patients studied was inadequate, the patients were too carefully selected, the protocols were too rigorous relative to routine medical practice, the treatment period was too short and/ or intermediary evaluation criteria were used.The information collected during phase III is by definition limited in time and space. The therapeutic norms (particularly the indication and dosage) established for a small homogeneous population will be extrapolated in phase IV to all patients regardless of their social or pathologic situation. Thus, these patients will differ from those of phase III.

In practice, disease is heterogeneous because it occurs within a non-uniform genetic inheritance and environment. Thus, the indication and dosage need to be adapted. It is necessary to carry out specific studies in subpopulations to which the prescribing physician can refer in order to define the safest conditions for application and to ensure that therapeutic trials are as plausible as possible. These subpopulations can be defined as a function of age, sex, ethnic origin and morphology. Age can clearly modify the pharmacokinetic and pharmacodynamic constants of a drug. The pharmacokinetic behavior of men and women of the same age can differ greatly, and the same is true for the pathology and the pharmacodynamic response. The therapeutic response can also differ depending on the race. Finally, the pharmacokinetics of a product can be modified by obesity or by associated pathology. Renal or hepatic insufficiency can modify the pharmacokinetics of a drug and favor its accumulation, thereby inducing toxic manifestations.

The dosage proposed at drug registration is broad enough to be adjusted to the needs of different patients and will thus be gradually redefined and generally lowered (minimum effective dose). It is based on the pathology of the hospitalized patient 
who is usually severely afflicted, whereas the ambulatory patient often has a different and less threatening condition of a more chronic nature requiring an adjustment of the dosage.

Large, "practical" or streamlined trials (LSTs) are used to study the effectiveness and/or safety of medicines in real world settings with minimal study imposed interventions [2]. In everyday practice, a physician is often led to prescribe two or more drugs for patients. As the combinations are infinite and cannot be studied in advance, trials are necessary. On the whole, phase IV studies should search for the optimal conditions of drug use for individual application. Phase IV gives the opportunity to study drug interactions.

Another purpose of phase IV trials is to check carefully if a side effect is induced by pathology or by drug treating this illness that the case with depression. Sexual dysfunction commonly occurs with major depressive disorder but antidepressants like SSRIs decrease libido. It is possible to understand in drug responders what is linked to depression or to antidepressants [3].

Economic purposes: facilitating product promotion: Once drug registration has been achieved, the new product begins its commercial life in an environment that will probably change in scientific, sociocultural and economic terms. In fact, the knowledge of pathologies improves, prescription strategies are developed, and consumer needs change (contraception, prevention of menopause disorders, etc.). In this environment, it is necessary to obtain the longest possible life cycle for the drug, which implies the following goals for the pharmaceutical firm: Familiarizing the greatest number of physicians with the product from the moment it is launched on the market. The pharmaceutical firm needs to make physicians aware of the product and its optimal conditions for use. In particular, reliable information based on rigorous scientific study can assist physicians in making correct therapeutic decisions. A better knowledge of the drug and of the subject to whom it is to be administered will reduce the frequency of adverse events, many of which occur when a drug is prescribed in less than ideal circumstances

The pharmaceutical firm attempts to collect the maximum of quantitative and qualitative information on the therapeutic behavior of its new product, which is beneficial to the practitioner who of course has no experience in prescribing the drug.The practitioner is required to develop and improve his knowledge in order to maintain his therapeutic potential at the highest scientific level. Thus, he must determine the true value of the new drug within his "therapeutic arsenal."

\section{Different types of phase IV trials}

For a pharmaceutical firm, research in phase IV trials involves the medical and marketing departments and pharmacovigilance. It is thus possible to define three different types of activity in phase IV: clinical research, pharmacovigilance and seeding trials.

Clinical research: A manufacturer develops a research plan based on a line of inquiry and recruits investigators.In certain cases, a trial may be requested by a physician who during his professional activity is confronted with difficulties, limitations and failures concerning the drug or who feels that improvements are needed.The promoters of these studies may be public authorities, state health insurance offices, patient associations, groups of physicians, etc. In fact, few studies of this type are undertaken since budgeting is not provided.

Health research, including health outcomes and comparative effectiveness research, is on the cusp of a golden era of access to digitized real-world data, catalyzed by the adoption of electronic health records and the integration of clinical and biological information with other data. This era promises more robust insights into what works in health care. Several barriers, however, will need to be addressed if the full potential of these new data are fully realized; these will involve both policy solutions and stakeholder cooperation technologies [4].

Seeding trials: One of the objectives of phase IV is to promote the commercial development of a drug. Although the purpose of the trial may be to communicate concepts or knowledge about the new product, certain studies known as seeding trials serve essentially to familiarize physicians with the drug.These studies allow the physician to become accustomed to prescribing a recently approved drug, possibly in consideration of certain incentives. However, some clinical seeding trials are justifiable if they are scientifically serious and correctly monitored.

Prescription studies or pseudo-trials for commercial purposes: The real intention of a pharmaceutical firm in conducting a phase IV trial may be to ensure that physicians prescribe the new drug. This is often accomplished by compensating participating physicians (i.e.investigators) for the treatment of patients. The drug is in fact purchased on prescription by patients and reimbursed by the social security system, generating a certain activity.

Payment to the investigator can consist of fees that are often proportional to the number of packages of the product prescribed or take the form of benefits not directly related to medical practice. This activity may only appear to have a scientific objective. In this case, it is not a free therapeutic act or an instance of valid research but simply a means of transferring money from the pharmaceutical firm to the prescribing physician and then from the patient back to the firm via the pharmacy.

Unlike true phase IV studies monitored by clinical research assistants, these seeding trials often make use of medical representatives and entertainment events to promote the relation between the pharmaceutical firm and the physician.Although these types of trials are currently decreasing in number, the commercial development of a product is still quite conceivable as an objective. In fact, scientific knowledge, development and promotion are complementary objectives which can be achieved simultaneously.

\section{Conclusion}

Clinical trials constitute the gold standard to assess the efficacy and safety of new medicines. However, because they are conducted in standardized conditions far from the real world of prescription and use, discrepancies in patient selection or 
treatment conditions may alter both the effectiveness and risks. That is the case of clinical trials in pregnant women.Relatively few drugs, especially those recently approved by the US Food and Drug Administration, have published human pregnancy experience. Although all drugs contain animal reproduction data, these are usually not predictive of human risk.Clinical trials in pregnant women are rarely conducted because of ethical and legal concerns, and it may be many years before sufficient observational data are collected to guide clinical treatment decisions. Because many of these drugs will be used in pregnancy, human data are needed shortly after the drugs come to the market .Lack of human data leads to uncertainty over whether a drug can be safely prescribed for a pregnant patient. Unless there are compelling scientific and ethical reasons to exclude them, pregnant women should be included in phase IV clinical trials (postmarketing studies to obtain additional information, including the risks, benefits, and optimal use of a drug) [5].

Although we do not believe that real world trials will supplant conventional randomized controlled trials, properly designed real-world trials will enrich our understanding of the effectiveness of new health care interventions and better inform patients and health care providers alike [6].

\section{References}

1. Martin K, Bégaud B, Latry P, Miremont-Salamé G, Fourrier A,
Moore N. Differences between clinical trials and postmarketing use. Br J Clin Pharmacol. 2004;57(1):86-92. doi: 10.1046/j.13652125.2003.01953.x.

2. Kolitsopoulos FM, Strom B, Faich G, Eng SM, Kane JM, Reynolds $\mathrm{RF}$. Lessons learned in the conduct of a global, large simple trial of treatments indicated for schizophrenia. Contemp Clin Trials. 2013;34(2):239-247. doi: 10.1016/j.cct.2012.12.001.

3. Clayton AH, Gommoll C, Chen D, Nunez R, Mathews M. Sexual dysfunction during treatment of major depressive disorder with vilazodone, citalopram, or placebo: results from a phase IV clinical trial. Int Clin Psychopharmacol. 2015;30(4):216-223. doi: 10.1097/ YIC.0000000000000075.

4. Berger ML, Lipset C, Gutteridge A, Axelsen K, Subedi P, Madigan D. Optimizing the leveraging of real-world data to improve the development and use of medicines. Value Health. 2015;18(1):127130 .

5. Briggs GG, Polifka JE, Wisner KL, Gervais E, Miller RK, Berard A et al. Should pregnant women be included in phase IV clinical drug trials? Am J Obstet Gynecol. 2015;213(6):810-815. doi: 10.1016/j. ajog.2015.05.047.

6. Freemantle N, Strack T. Real-world effectiveness of new medicines should be evaluated by appropriately designed clinical trials. J ClinEpidemiol. 2010;63(10):1053-1058. doi: 10.1016/j. jclinepi.2009.07.013. 